Corps et culture

Numéro 3 | 1998

Sport et lien social

\title{
Mythologie de la solidarité sportive
}

\section{Betty Lefèvre}

\section{(2) OpenEdition}

Journals

Édition électronique

URL : http://journals.openedition.org/corpsetculture/776

DOI : 10.4000/corpsetculture.776

ISSN : $1777-5337$

\section{Éditeur}

Association Corps et Culture

Édition imprimée

Date de publication : 1 juin 1998

ISSN : 1268-5631

Référence électronique

Betty Lefèvre, "Mythologie de la solidarité sportive », Corps et culture [En ligne], Numéro 3 | 1998, mis en ligne le 11 octobre 2007, consulté le 08 septembre 2020. URL : http://journals.openedition.org/ corpsetculture/776 ; DOI : https://doi.org/10.4000/corpsetculture.776

Ce document a été généré automatiquement le 8 septembre 2020

(c) tous droits réservés 


\title{
Mythologie de la solidarité sportive
}

\author{
Betty Lefèvre
}

1 Le récit qui s'élabore sur le sport de compétition, en renouvelle sans cesse l'auréole mythique et, par ailleurs, le fantasme collectif d'anomie stigmatise les angoisses d'une société en panne de communication, de valeurs, de croyances et de liens de solidarité.

2 Ainsi dit-on du sport qu'il est créateur et/ou renforçateur du lien social. Qu'est ce qui fonde cette affirmation? De quelle nature est ce lien ? Cela ne nous renverrait-il pas à une pensée strictement fonctionnaliste visant à prouver que le sport, fait social, développe une fonction spécifique, agrégative et génératrice de socialisation?

3 Si le sport est plus socialisant que d'autres pratiques, doit-on le ramener à l'émergence d'interdépendances organiques comme le fit E. Durkheim (1926) ? En cela représente-til une structure de solidarité ?

4 Enfin, si on avance que la pratique sportive est surtout constitutive du lien avec une corporéité, en quoi cette reliance est-elle transférable dans l'espace social ?

5 L'hypothèse proposée dans cette réflexion est que les formes de " socialité » attachées au sport se construisent autour de valeurs antinomiques, paradoxales. J'emprunterai à M. Maffesoli sa définition de la socialité comme étant: « l'expression quotidienne de la solidarité de base, le sociétal en acte» (M. Maffesoli, 1985, p. 13).

6 Les imaginaires contradictoires qui entourent le sport réinventent, reconstruisent la réalité autour d'un processus dynamique de mythification. Rappelons qu'au sens traditionnel du terme, le mythe se définit comme un récit, souvent héroïque, dont la fonction essentielle est de proposer un essai d'explication et/ou de mise en ordre du monde. Ce type de savoir sur les mythes est-il pertinent pour saisir le réel ? Utilisant la sémiologie de $\mathrm{F}$ de Saussure, R. Barthes, dans une tentative de saisie holistique du donné sociétal, propose de considérer le mythe, comme un " métalangage, c'est-à-dire une seconde langue dans laquelle on parle de la première » (R. Barthes, $1957: 200$ ). Notre réflexion sur l'objet mythique sport se fera à partir de cette acceptation élargie du terme.

7 D'autre part, face à cette réalité sportive complexe confrontée à ses mythes, nous tenterons une approche compréhensive qui s'appuiera largement sur les différents 
ouvrages de M. Maffesoli, tant son attention à développer une sociologie du quotidien, de l'ordinaire, du sensible parait incontournable pour repérer ce qui fait sens dans nos pratiques actuelles. C'est à partir de ce regard lucide et généreux que l'on s'essaiera à décliner cette création mythique de la solidarité par le sport.

8 Rappelons succinctement que tout processus de socialisation s'élabore à partir de l'intégration des règles du groupe, d'une acceptation des normes et des lois de la culture d'appartenance. L'individu social intériorise de façon Plus ou moins consciente des interdits, des modèles de comportement, des valeurs que les structures sociales (via la famille, l'école, le travail, etc.) lui imposent.

9 En cette fin de siècle, les éléments constitutifs du lien social se sont effilochés certains y voient, au travers de la montée de l'individualisme, les signes d'une pathologie du social et d'autres, comme $M$. Maffesoli, y repèrent l'émergence " d'une socialité à dominante empathique "(M. Maffesoli, 1988: 23) c'est-à-dire des regroupements sociaux plus aléatoires, plus éphémères, plus " topiques " et qui renvoient essentiellement au désir d'éprouver des émotions ensemble.

10 Pour répondre à ce baroquisme de nos rapports sociaux, le sport parmi d'autres objets culturels, devient synonyme de valeurs refuges. C'est ainsi, qu'à travers l'analyse discursive des récits sportifs s'élabore un véritable conservatoire de ses vertus " natives " : la culture sportive repose sur des postulats, et des prénotions constitutives d'un large spectre mythique. Le sport, quoi qu'il arrive, véhicule une image de pureté. C'est l'école de la vie, de l'amitié. C'est le lieu où les hiérarchies sociales s'estompent voire disparaissent, où les nations se réconcilient. Bref, le sport rassemble en exaltant altruisme et solidarité. La grande famille du club et/ ou de l'équipe en sport collectif est souvent proposée comme une forme de substitution efficace face au démantèlement des traditions familiales, ce qui n'est pas sans nous suggérer l'idée d'un lien organique, biologique associé au grand mythe des solidarités de sang,

11 Même si on sait, grâce aux travaux de P. Parlebas (1987 : 159-167) que la confusion fréquente entre faits d'interaction et faits de socialisation génère des amalgames sur le caractère " plus socialisateur » des sports collectifs, il n'en reste pas moins vrai que le modèle sportif implique un processus strict d'obéissance aux règlements, la référence à l'ordre, à la hiérarchie, la valorisation d'un corps outil, maîtrisé, efficient, rentable. Ce qui reprend en écho les indicateurs de l'incorporation idéale des valeurs sociales dominantes et présente l'activité sportive comme un méta-projet visant à renforcer l'ordre social.

12 Or dans un même temps et par un assemblage que l'on pourrait peut-être qualifier de dualisme schématique, le sport se fonde sur un mécanisme de séparation, d'individuation radicale. Toute excellence renvoie à la sélection, à l'exclusion. Le gagnant, le vainqueur, l'unique va prévaloir sur le tout. Ce qui est à l'oeuvre dans tout jeu sportif, c'est d'abord une praxis d'opposition, une culture corporelle qu'avec R. Caillois (1967) on peut qualifier d'agonistique et où prédomine la relation duelle.

On peut avancer que c'est dans cette dynamique entre reliance et séparation que toute pratique sportive trouve à la fois sa légitimité et la permanence de son aura mythique. Le sport ne représente-t-il pas cet espace paradoxal décrit par G. Simmel dans son article, Pont et portes où il constate : "Dans un sens immédiat aussi bien que symbolique, et corporel aussi bien que spirituel, nous sommes à chaque instant ceux qui séparent le relié ou qui relient le séparé parce que l'homme est l'être de liaison qui 
doit toujours séparer et qui ne peut relier sans avoir séparé " (G. Simmel, 1988: 159-166). Ceci l'amène à considérer le chemin comme une grande performance humaine dont le pont est le mode le plus achevé puisque c'est un objet de jonction entre deux lieux initialement séparés. De la même façon pourrait-on écrire que le sport établit des ponts, peut-être involontaires mais qui attachent les hommes entre eux, et des portes qui les séparent en instaurant la destruction symbolique de l'autre. C'est ce mouvement dialectique entre imaginaire d'alliance et imaginaire d'opposition qui fait sens pour saisir nos rituels sportifs.

Une première déclinaison autour de cette métaphore des ponts et des portes du sport empruntera au basket de rue qui, bien que constitutif d'un détournement de la culture sportive, peut nous permettre de repérer la mise en oeuvre du mythe de la socialisation par le sport. Qu'est-ce qui se joue sur les playground? Ces espaces constituent-ils les nouveaux remèdes aux problèmes endémiques de nos cités ? Est-ce là que se régénère notre capacité à la reliance ou que se restructure notre « être ensemble »? Quelles sont les attentes poursuivies par les auteurs producteurs du phénomène?

Il semblerait que la pratique du basket de rue s'organise autour de quatre principes :

- Pas de règle (chaque playground a les siennes)

- Pas d'arbitre

- Pas d'horaire

- Pas d'entraîneur

Comment lire ce comportement de refus ? Brièvement, il semble que deux pistes interprétatives sont à considérer.

D'une part, les basketteurs de rue nous donnent à voir leur peur de l'intégration sociale et/ou leur volonté infra consciente de s'exclure. A ce sujet, M. Fize écrit : «Quand on reste entre soi, sans adulte, comme dans les sports de rue, on ne grandit pas, cela révèle l'angoisse d'une génération pour le passage à l'état adulte ou le contact avec la vie sociale. » (M. Fize, 1994)

D'autre part, on peut penser que ces adolescents stigmatisent les grandes institutions sociales (l'école mais aussi les fédérations sportives) dont la légitimation repose essentiellement sur une logique d'ordre, sur un espace normé et un temps mesuré, socialisant par excellence. Enfin ils dénoncent à leur façon le contexte de rejet, d'exclusion voire de mépris qui les entoure.

Or très curieusement, éducateurs et politiques semblent ignorer cette prise de distance avec le sport officiel, cet avertissement lancé à une société de la " gagne à tout prix » pour s'attacher uniquement à la vertu intégratrice de cette pratique ludo-sportive. Les jeunes du basket de rue revendiquent le droit à un usage "détourné » d'un sport institué qui, en participant à l'intégration des règles du social, génère souvent inégalité et exclusion. Car s'ils proposent un autre usage, une autre interprétation du sport, c'est qu'ils en ont peut-être repéré les mécanismes d'assujettissement au sens où l'entend $M$. Foucault de rapport de docilité-utilité des corps (M. Foucault, 1977 : 139). Doit-on rappeler que le sport de compétition, le basket-ball entre autres, repose sur une logique économique de la rentabilité et de l'efficience, celle du marché de la concurrence ? Les jeunes acteurs de basket de rue, tout en étant fascinés par le modèle " académique » du basket-ball américain, affirment leur autonomie en le réécrivant et en échappant aux différents programmes qui leur sont appliqués. 
21 S'ils ne génèrent pas une contre-culture, on pourrait écrire en reprenant les travaux de M. Maffesoli (1988) que le basket de rue se construit autour d'une tribu, le groupe se solidarisant autour d'un terrain/territoire et une reconnaissance qui s'appuie sur un imaginaire d'identification au jeu des stars du basket-ball américain.

Ces basketteurs urbains, outre l'usage détourné qu'ils font du sport légitimé, invitent à penser leur pratique comme un espace paradoxal où les imaginaires se confrontent. D'une part, ils sollicitent un imaginaire agonal du combat et de l'opposition qu'ils érigent en art compétitif. Pour retrouver une estime de soi, pour se réhabiliter, ces jeunes se lancent des défis au travers de différentes pratiques dont certaines ne sont pas sans risque. Comme le précise B. Jeu (Il 987 : 187-190), le sport établit alors une dialectique entre Eros et Thanatos et nous met à l'écoute de ses résonances mythologiques (triomphe de la vie sur la mort, dépassement de soi, soumission à l'épreuve, consécration de l'exploit. D'autre part, ils recréent un sport relevant d'un imaginaire d'alliance, voire d'une nostalgie du lien rompu et qui s'exprime par l'émotion véhiculée lors de ses grandes mises en spectacle : le sport, transporteur d'émotion, «forme sensible de la vie sociale » dirait P. Sansot (Il 986 : 63-103), revivifie notre socialité.

Dans ce rapport au corps s'instaure une esthétique du "vivre ensemble », où s'éprouve l'effervescence émotionnelle de la turbulence festive. C'est dans cet espace que se donne à voir une " éthique de l'esthétique » au sens maffessolien du terme, c'est-à-dire qu'il s'agit en premier d'éprouver en commun, de partager des émotions. En bref, écrit M. Maffesoli : « le lien social devient émotionnel » (M. Maffesoli, 1990 : 13).

24 Face à la désintégration du tissu social, on confère au sport via les corps sensibles, la tâche de grand tisserand du lien social, de grand réformateur de la solidarité rompue.

En résumé, le postulat à priori des vertus socialisatrices du sport, comme école d'éducation à la vie sociale, est revisité au sein de pratiques plus ou moins informelles refusant l'institutionnalisation et où se conjuguent passions, sentiments et affects. C'est là qu'apparaît ce lien social impensé, « résiduel » écrirait V Pareto (1965), et pourtant fondamentalement constitutif du jeu sociétal.

Notre deuxième déclinaison de la mythologie de la solidarité sportive va s'attacher, dans un monde occidental saturé de rationalité, à repérer l'irruption d'une pensée magique, d'une résurgence du religieux. Car la notion de lien renvoie d'abord au religieux, la religion étant, au-delà de toutes subtilités théologiques, étymologiquement ce qui relie.

27 Or, si les grandes religions traditionnelles se sont affaiblies, d'autres formes de religiosités, de rapport au sacré, se sont imposées. Ainsi, J. Birouste note que : "Notre $\mathrm{XX}^{\mathrm{e}}$ siècle finissant convertit une large part de sacré dans les rituels, les solennités et les parades sportives » (J. Birouste, $1991: 183$ ). Face à ce grand désarroi des valeurs, peut-on dire que le sport fonctionne comme substitut à nos pertes de croyances ? Comment se concrétise cette religiosité du sport?

Sans avoir la prétention à l'exhaustivité, on peut avancer que les grands événements sportifs réhabilitent le religieux en imposant par exemple une structuration originale et profane du temps. G. Vigarello, dans Le temps du sport, démontre que le sport instaure une suspension du quotidien voire une rupture comme l'ont toujours fait les fêtes religieuses (G. Vigarello, 1995 : 209-210). 
tre part, ce qui fait que l'on s'adonne à un sport relève d'une pluri-causalité parm lesquelles on peut s'attacher au lien plus ou moins explicite que la pratique permet d'entretenir avec le corps.

$\mathrm{Au}$ travers des rituels sportifs, la relation à la corporéité devient envahissante, fusionnelle. A ce sujet J. Duvignaud écrit : «Que ce passe-t-il lorsque se composent ces complicités de fête ? Participation, ouverture des consciences les unes aux autres, fusion quasi mystique - ce sont les seuls termes dont nous disposions " (J. Duvignaud, $1986: 200)$.

C'est pourquoi on peut dire que le sport renvoie au mystique et à ce que M. Maffesoli appelle « la transcendance immanente » (M. Maffesoli, 1985 : 119), le sportif apparaissant à la fois comme symbole de vie transcendée, comme idéal de transcendance et de maîtrise de soi. Par la désignation d'un vainqueur la tragédie sportive au cours de ses spectacles renoue avec l'ordalie, avec l'acceptation d'un jugement divin mais aussi avec ce que Bernard Jeu nomme « la valeur hiérogamique du sport » ce mariage toujours répété de l'homme et de ses dieux.

En conclusion, on pourrait écrire que le sport fonctionne comme une légende il n'est ni synchronique ni diachronique, il est anachronique, il est opposition et alliance, pont et porte, profane et sacré à la fois. Il représente bien ce mouvement, ce dynamisme irréductible du corps social constitutif du vivant et de la socialité.

34 Certes la réalité du sport ne peut se réduire à l'expression de ces « dialogiques » mais cette logique antinomique est à prendre en compte comme une reconnaissance de la complexité du monde, de l'ambivalence et ne manque pas d'ouvrir les voies d'une véritable phénoménologie compréhensive des pratiques sportives et de ses constructions mythiques.

Ecoutons encore cette respiration sociale dont M. Maffesoli se fait l'écho

« On commence à se rendre compte maintenant que le vaste processus de démythologisation, et ce dans tous les domaines, prive le corps (social, cosmique) de ses défenses naturelles. En effet, quand il y a pluralité de valeurs (polythéisme), il y a neutralisation réciproque, relativisation. Ce jeu antinomique permet la perdurance de l'Etre. Faute d'une telle antinomie c'est le rouleau compresseur d'une idéologie, d'un système d'idées qui prévaut avec, régulièrement, l'explosion de ce que l'on appelle le retour du refoulé. Penser la contradiction c'est à cet égard faire preuve de santé » (M. Maffesoli, $1985: 116)$.

\section{BIBLIOGRAPHIE}

Barthes R.(1 957) Mythologies. Paris, Seuil.

Birouste J. (1991) Actes du colloque : Anthropologie du sport, Perspectives critiques,.Paris, Coédition ANDSHA, Quels corps?

Caillois R. (1967) Les jeux et les hommes. Paris, Gallimard,

Durkheim E. (1926) De la division du travail, Paris, Edition F Alcan

Corps et culture, Numéro $3 \mid 1998$ 
Duvignaud J. (1986) La solidarité. Paris, Fayard,

Fize M. (1994) Le peuple adolescent. Paris, Julliard,

Foucault M. (1977) Surveiller et punir. Paris, Gallimard.

Jeu B. (1987) Analyse du sport. Paris, PUF

Maffesoli M. (1982) L'ombre de Dionysos: contribution à une sociologie de l'orgie, Paris, Librairie des Méridiens.

Maffesoli M. (1985) La connaissance ordinaire. Précis de sociologie compréhensive, Paris, Librairie des Méridiens.

Maffesoli M. (1988) Le temps des tribus. Le déclin de l'individualisme dans la société de masse.

Maffesoli M. (1990) Au creux des apparences. Pour une éthique de l'esthétique. Paris, Pion.

Pareto V. (1965), Traité de sociologie générale. Genève, Droz.

Parlebas P. (1987) Sport en jeux. Paris, C. E.M.E.A.

Simmel G. (1988) La tragédie de la culture, Paris, Ed. Rivages.

Sansot P. (1986) Les formes sensibles de la vie sociale. Paris, RU.F.

Vigarello G. (1995) Le temps du sport. A. CORBIN L'avènement des loisirs, 1850, 1960. Paris, Ed. Aubier.

\section{RÉSUMÉS}

Dans une société encombrée par l'omnipotence de ses institutions et l'omniprésence de la performativité économique et technique, la quête du LIEN, de la reliance, s'insinue comme un parfum étrange au sein de notre paysage social, inquiet de la perte des valeurs qui fondent le collectif. Le sport, pratique culturelle et sociale, n'échappe pas à cette recherche et développe, au travers de ses politiques et de ses récits, le thème récurrent de la grande solidarité sportive. A contrario, on peut noter que la logique interne de toute situation de compétition sportive met l'accent sur ce qui sépare, ce qui classe et distingue.

In a society overburdenet with omniprotent institutions and omnipresent positivist knowledge, a society that is losing the values by which it used to be defined, the search of the LINK, of the relationship, is creeping into the social landscape like some unusual fragance in the air. Sports, as social and cultural activities, are also concerned by this search. Through their policies and the way they are dealt, they develop the recurrent theme of the great sporting solidarity. A contrario, we can reckon that the internal rationale of any kind of sport competition emphasizes separation, classification, distinction. To overcome this paradox and in reference to Michel Maffesoli's sociology "vagabonde", we'll try to see how this myth of a sportingsolidarity eventually contributes to the expression of the " 0 us ".

\section{INDEX}

Keywords : sport, solidarity, myth, sociology of feeling

Mots-clés : sociologie du sensible, solidarité, mythe 
AUTEUR

BETTY LEFÈVRE

Faculté des Sciences du Sport et de l'Education Physique, Université de Rouen, Centre d'Etudes des Transformations des Activités Physiques et Sportives (CETAPS) 